\title{
Further results on Cauchy tensors and Hankel tensors
}

\author{
Haibin Chen ${ }^{\mathrm{a}, 1, *}$, Guoyin $\mathrm{Li}^{\mathrm{b}, 2}$, Liqun $\mathrm{Qi}^{\mathrm{a}, 3}$ \\ a Department of Applied Mathematics, The Hong Kong Polytechnic University, Hung Hom, Kowloon, Hong Kong \\ ${ }^{\mathrm{b}}$ Department of Applied Mathematics, University of New South Wales, Sydney 2052, Australia
}

\section{A R T I C L E I N F O}

\section{MSC:}

90C 30

15 A06

\section{Keywords:}

Generalized Cauchy tensor

SOS tensor

Hankel tensor

Positive semi-definiteness

$\mathrm{H}$-eigenvalue

\begin{abstract}
A B S T R A C T
In this article, we present various new results on Cauchy tensors and Hankel tensors. We first introduce the concept of generalized Cauchy tensors which extends Cauchy tensors in the current literature, and provide several conditions characterizing positive semi-definiteness of generalized Cauchy tensors with nonzero entries. Furthermore, we prove that all even order generalized Cauchy tensors with positive entries are completely positive tensors, which means every such that generalized Cauchy tensor can be decomposed as the sum of nonnegative rank-1 tensors. We also establish that all the H-eigenvalues of nonnegative Cauchy tensors are nonnegative. Secondly, we present new mathematical properties of Hankel tensors. We prove that an even order Hankel tensor is Vandermonde positive semi-definite if and only if its associated plane tensor is positive semi-definite. We also show that, if the Vandermonde rank of a Hankel tensor $\mathcal{A}$ is less than the dimension of the underlying space, then positive semi-definiteness of $\mathcal{A}$ is equivalent to the fact that $\mathcal{A}$ is a complete Hankel tensor, and so, is further equivalent to the SOS property of $\mathcal{A}$. Thirdly, we introduce a new class of structured tensors called Cauchy-Hankel tensors, which is a special case of Cauchy tensors and Hankel tensors simultaneously. Sufficient and necessary conditions are established for an even order Cauchy-Hankel tensor to be positive definite.
\end{abstract}

(c) 2015 Elsevier Inc. All rights reserved.

\section{Introduction}

Let $\mathbb{R}^{n}$ be the $n$ dimensional real Euclidean space. Denote the sets of all natural numbers by $\mathbb{N}$. Suppose $m, n \in \mathbb{N}, m, n \geq 2$ and denote $[n]=\{1,2, \ldots, n\}$. It should be noted in advance, we always consider real order $m$ dimension $n$ tensors in this paper.

Tensors (or sometimes called hypermatrices) are the multi-array extensions of matrices. It was recently demonstrated in [13] that most of the problems associated with tensors are, in general, NP-hard. So, it motivates researchers to study tensors with special structure i.e. structured tensors. In the last two or three years, a lot of research papers on structured tensors appeared [3,4,8,9,12,20,28,29,33,37-39,41]. These include M-tensors, circulant tensors, completely positive tensors, Hankel tensors, Hilbert tensors, P-tensors, B-tensors and Cauchy tensors. Many interesting properties and meaningful results of structured tensors have been discovered. For instance, spectral properties of structure tensors, positive definiteness and semi-definiteness of structured tensors were established. Furthermore, some practical applications of structured tensors were studied such as application in

\footnotetext{
* Corresponding author. Tel.: +86 6333981571.

E-mail addresses: chenhaibin508@163.com (H. Chen), g.li@unsw.edu.au (G. Li), maqilq@polyu.edu.hk (L. Qi).

1 This author's work was supported by the Natural Science Foundation of China (11171180).

2 This author's work was partially supported by Australian Research Council.

${ }^{3}$ This author's work was supported by the Hong Kong Research Grant Council (Grant No. PolyU 502111, 501212, 501913 and 15302114).
} 
stochastic process and data fitting $[4,9]$. Very recently, authors of [20] studied SOS-Hankel tensors and applied them to the positive semi-definite tensor completion problem.

Among the various structured tensors we mentioned above, there are two particular interesting classes: Cauchy tensors and Hankel tensors. The symmetric Cauchy tensors were defined and analyzed in [3]. In the following discussion, we simply refer it as Cauchy tensors instead of symmetric Cauchy tensors. One of the nice properties of a Cauchy tensor is that its positive semidefiniteness (or positive definiteness) can be easily verified by the sign of the associated generating vectors. In fact, it was proved in [3] that an even order Cauchy tensor is positive semi-definite if and only if each of entries of its generating vector is positive, and an even order Cauchy tensor is positive definite if and only if each entries of its generating vector is positive and mutually distinct.

Hankel tensors arise from signal processing and data fitting [1,9,25]. As far as we know, the definition of Hankel tensor was first introduced in [25]. Recently, some easily verifiable structured tensors related to Hankel tensors were also introduced in [28]. These structured tensors include strong Hankel tensors, complete Hankel tensors and the associated plane tensors that correspond to underlying Hankel tensors. It was proved that if a Hankel tensor is copositive or an even order Hankel tensor is positive semi-definite, then the associated plane tensor is copositive or positive semi-definite respectively [28]. Furthermore, results on positive semi-definiteness of even order strong and complete Hankel tensors were given. However, the relationship between strong Hankel tensors and complete Hankel tensors was not provided in [28]. Later, in [20], it was shown that complete Hankel tensors are strong Hankel tensors; while the converse is, in general, not true.

In this paper, we will provide further new results for Cauchy tensors and Hankel tensors which complements the existing literature. The remainder of this paper is organized as follows. In Section 2, we will first recall some basic notions of tensors are given such as $\mathrm{H}$-eigenvalues, Z-eigenvalues and positive semi-definite tensors. We will also introduce the notion of Vandermonde positive semi-definite tensors, which is a special class of positive semi-definite tensors.

In Section 3, we will first introduce the generalized Cauchy tensors which is an extension of the Cauchy tensors in the literature. Then, we provide complete characterization for positive semi-definite generalized Cauchy tensors with nonzero entries. We also present sufficient and necessary conditions guaranteeing an even order generalized Cauchy tensor with nonzero entries to be completely decomposable. After that, it is proven that all even order generalized Cauchy tensors with positive entries are SOS (sum-of-square) tensors if and only if they are completely positive tensors, which means every generalized Cauchy tensor with positive entries can be written as a sum of nonnegative rank-1 tensors. Furthermore, we prove that the Hadamard product of two positive semi-definite Cauchy tensors are still positive semi-definite tensors. And the nonnegativity for H-eigenvalues of nonnegative Cauchy tensors are testified.

In Section 4, we provide further new properties of Hankel tensors. We prove that the associated plane tensor of an even order Hankel tensor is positive semi-definite if and only if the Hankel tensor is Vandermonde positive semi-definite. Using this conclusion, we give an example to show that, for higher dimensional Hankel tensors, the associated plane tensor is positive semi-definite but the Hankel tensor failed. We also show that, if the Vandermonde rank of a Hankel tensor $\mathcal{A}$ is less than the dimension of the underlying space, then positive semi-definiteness of $\mathcal{A}$ is equivalent to the fact that $\mathcal{A}$ is a complete Hankel tensor, and so, is further equivalent to the SOS property of $\mathcal{A}$.

In Section 5, we introduce Cauchy-Hankel tensors, which are natural extensions of Cauchy-Hankel matrices. The class of Cauchy-Hankel tensors is a subset of Cauchy tensors [3] and Hankel tensors [9,20,28] simultaneously. We provide a checkable sufficient and necessary condition for an even order Cauchy-Hankel tensor to be positive definite. We also show that an even order Cauchy-Hankel tensor is positive semi-definite if and only if the associated homogeneous polynomial is strict monotonically increasing on the nonnegative orthant $\mathbb{R}_{+}^{n}$. Some final remarks are provided in Section 6.

Before we end the introduction section, let us make some comments on the symbols that will be used throughout this paper. Vectors are denoted by italic lowercase letters i.e. $x, y, \ldots$, and matrices are denoted by capital letters $A, B, \ldots$. Suppose $e \in \mathbb{R}^{n}$ be all one vectors and let $e_{i}$ denotes the $i$ th unite coordinate vector in $\mathbb{R}^{n}$. We use bold letters $\mathbf{0} \in \mathbb{R}^{n}$ to denote zero vector. Tensors are written as calligraphic capitals such as $\mathcal{A}, \mathcal{T}, \ldots$ Let $\mathcal{I}$ denote the real identity tensor. For $x=\left(x_{1}, x_{2}, \ldots, x_{n}\right)^{T}, y=$ $\left(y_{1}, y_{2}, \ldots, y_{n}\right)^{T} \in \mathbb{R}^{n}$, then $x \geq y(x \leq y)$ means $x_{i} \geq y_{i}\left(x_{i} \leq y_{i}\right)$ for all $i \in[n] . x^{[m]}$ is defined by $\left(x_{1}^{m}, x_{2}^{m}, \ldots, x_{n}^{m}\right)^{T}$.

\section{Preliminaries}

A real tensor with order $m$ and dimension $n$ is defined by $\mathcal{A}=\left(a_{i_{1} i_{2} \ldots i_{m}}\right), i_{j} \in[n], j \in[m]$. If the entries $a_{i_{1} i_{2} \ldots i_{m}}$ are invariant under any permutation of the subscripts, then tensor $\mathcal{A}$ is called symmetric tensor. Let $x=\left(x_{1}, x_{2}, \ldots, x_{n}\right)^{T} \in \mathbb{R}^{n}$. The two forms below will be used in the following analysis frequently:

$$
\begin{aligned}
\mathcal{A} x^{m-1} & =\left(\sum_{i_{2}, i_{3}, \ldots, i_{m}=1}^{n} a_{i i_{2} \ldots i_{m}} x_{i_{2}} \ldots x_{i_{m}}\right)_{i=1}^{n} ; \\
\mathcal{A} x^{m} & =\sum_{i_{1}, i_{2}, \ldots, i_{m}=1}^{n} a_{i_{1} i_{2} \ldots i_{m}} x_{i_{1}} x_{i_{2}} \ldots x_{i_{m}} .
\end{aligned}
$$

Denote $\mathbb{R}_{+}^{n}=\left\{x \in \mathbb{R}^{n} \mid x \geq \mathbf{0}\right\}$. If $\mathcal{A} x^{m} \geq 0$ for all $x \in \mathbb{R}_{+}^{n}$, then $\mathcal{A}$ is called copositive. An even order $\mathrm{m}$ dimension $\mathrm{n}$ tensor $\mathcal{A}$ is called positive semi-definite if for any vector $x \in \mathbb{R}^{n}$, it satisfies $\mathcal{A} x^{m} \geq 0$. Tensor $\mathcal{A}$ is called positive definite if $\mathcal{A} x^{m}>0$ for all nonzero vectors $x \in \mathbb{R}^{n}$. From the definition, it is easy to see that, for a positive semi-definite tensor, its order $m$ must be an even 
number. Therefore, in the following analysis, we always assume the order of the tensor is even when we consider a positive semi-definite tensor.

We call $u \in \mathbb{R}^{n}$ a Vandermonde vector if $u=\left(1, \mu, \mu^{2}, \ldots, \mu^{n-1}\right)^{T} \in \mathbb{R}^{n}$ for some $\mu \in \mathbb{R}$. If $\mathcal{A} u^{m} \geq 0$ for all Vandermonde vectors $u \in \mathbb{R}^{n}$, then we say that tensor $\mathcal{A}$ is Vandermonde positive semi-definite. It's obvious that positive semi-definite tensors are always Vandermonde positive semi-definite, but not vice versa.

Next, we recall the definitions of eigenvalues of tensors.

Definition 2.1. Let $\mathcal{A}$ be a symmetric tensor with order $m$ and dimension $n$. We say $\lambda \in \mathbb{R}$ is a $Z$-eigenvalue of $\mathcal{A}$ and $x \in \mathbb{R}^{n} \backslash\{\mathbf{0}\}$ is an $Z$-eigenvector corresponding to $\lambda$ if $(x, \lambda)$ satisfies

$$
\left\{\begin{array}{l}
\mathcal{A} x^{m-1}=\lambda x \\
x^{T} x=1
\end{array}\right.
$$

Moreover, we say $\lambda \in \mathbb{R}$ is an $H$-eigenvalue of $\mathcal{A}$ and $x \in \mathbb{R}^{n} \backslash\{\mathbf{0}\}$ is an $H$-eigenvector corresponding to $\lambda$ if $(x, \lambda)$ satisfies

$$
\mathcal{A} x^{m-1}=\lambda x^{[m-1]},
$$

where $x^{[m-1]}=\left(x_{1}^{m-1}, \ldots, x_{n}^{m-1}\right)^{T} \in \mathbb{R}^{n}$.

The definitions of $Z$-eigenvalue and $H$-eigenvalue were introduced by Qi in [27]. Independently, Lim [24] also gave the definitions via a variational approach and established an interesting Perron-Frobenius theorem for tensors with nonnegative entries. From [27] and [5], both Z-eigenvalues and $H$-eigenvalues for an even order symmetric tensor always exist. Moreover, from the definitions, we can see that finding an $\mathrm{H}$-eigenvalue of a symmetric tensor is equivalent to solving a homogeneous polynomial equation while calculating a $Z$-eigenvalue is equivalent to solving nonhomogeneous polynomial equations. In general, the behaviors of $Z$-eigenvalues and $H$-eigenvalues can be quite different. For example, a diagonal symmetric tensor $\mathcal{A}$ has exactly $n$ many $H$-eigenvalues and may have more than $n$ Z-eigenvalues (for more details see [27]). Recently, a lot of researchers have devoted themselves to the study of eigenvalue problems of symmetric tensors and have found important applications in diverse areas including spectral hypergraph theory [7,23], dynamical control [31], medical image science [22,32] and signal processing [17].

\section{SOS properties and complete positivity of even order generalized Cauchy tensors}

Symmetric Cauchy tensors was first studied in [3]. Some checkable sufficient and necessary conditions for an even order symmetric Cauchy tensor to be positive semi-definite or positive definite were provided in [3], which extends the matrix cases established in [10].

Definition 3.1 [3]. Let $c=\left(c_{1}, c_{2}, \ldots, c_{n}\right)^{T} \in \mathbb{R}^{n}$. Let a real tensor $\mathcal{C}=\left(c_{i_{1} i_{2} \ldots i_{m}}\right)$ be defined by

$$
c_{i_{1} i_{2} \ldots i_{m}}=\frac{1}{c_{i_{1}}+c_{i_{2}}+\cdots+c_{i_{m}}}, \quad j \in[m], i_{j} \in[n] .
$$

Then, we say that $\mathcal{C}$ is a symmetric Cauchy tensor with order $m$ and dimension $n$. The corresponding vector $c \in \mathbb{R}^{n}$ is called the generating vector of $\mathcal{C}$.

Now, given two vectors $c=\left(c_{1}, c_{2}, \ldots, c_{n}\right)^{T}, d=\left(d_{1}, d_{2}, \ldots, d_{n}\right)^{T} \in \mathbb{R}^{n}$. Consider the generalized Cauchy tensor $\mathcal{C}=$ $\left(c_{i_{1} i_{2} \ldots i_{m}}\right)$ with order $m$ dimension $n$, where

$$
c_{i_{1} i_{2} \ldots i_{m}}=\frac{d_{i_{1}} d_{i_{2}} \ldots d_{i_{m}}}{c_{i_{1}}+c_{i_{2}}+\cdots+c_{i_{m}}}, i_{j} \in[n], j \in[m] .
$$

For the sake of simplicity, we call vectors $c, d$ the generating vectors of the generalized Cauchy tensor $\mathcal{C}$. In the special case when $d_{i}=1, i \in[n]$, a generalized Cauchy tensor reduces to a Cauchy tensor defined in Definition 3.1. In the case when $m=2$, a generalized Cauchy tensor collapses to a symmetric generalized Cauchy matrix [26]. We also note that every rank-one tensor with the form $u^{m}$ for some $u \in \mathbb{R}^{n}$ is, in particular, a generalized Cauchy tensor.

Define Cauchy tensor $\overline{\mathcal{C}}=\left(\bar{c}_{i_{1}, i_{2}, \ldots i_{m}}\right)$ where

$$
\bar{c}_{i_{1}, i_{2}, \ldots i_{m}}=\frac{1}{c_{i_{1}}+c_{i_{2}}+\cdots+c_{i_{m}}}, i_{j}=1, \ldots, n, j=1, \ldots, m .
$$

It is easy to see for any $x \in \mathbb{R}^{n}$, we have

$$
\mathcal{C} x^{m} \equiv \overline{\mathcal{C}} y^{m},
$$

where $y \in \mathbb{R}^{n}$ with $y_{i}=d_{i} x_{i}$ for $i=1, \ldots, n$. By Theorems 2.1 and 2.3 of [3], one may easily conclude that the generalized Cauchy tensor $\mathcal{C}$ is positive semi-definite if and only if $d_{i}=0, c_{i} \neq 0$ or $d_{i} \neq 0, c_{i}>0, i \in[n]$ and $\mathcal{C}$ is positive definite if and only if $c_{1}, c_{2}, \ldots, c_{n}$ are positive real number and mutually distinct, and $d_{i} \neq 0, i=1, \ldots, n$.

In this section, we mainly characterize SOS (sum-of-squares) properties and completely positiveness of even order generalized Cauchy tensors with nonzero entries. Before giving the main results, we briefly recall the definitions of SOS tensors and completely positive tensors. 
SOS tensors are first defined in [14]. The definition of SOS tensors relies on the celebrated concept of SOS polynomials, which is a fundamental concept in polynomial optimization theory $[14,15,18,19,34]$. Assume $\mathcal{A}$ is an order $m$ dimension $n$ symmetric tensor. Let $m=2 k$ be an even number. If

$$
f(x)=\mathcal{A} x^{m}, x \in \mathbb{R}^{n}
$$

can be decomposed to the sum of squares of polynomials of degree $k$, then $f$ is called a sum-of-squares (SOS) polynomial, and the corresponding symmetric tensor $\mathcal{A}$ is called an SOS tensor [14]. From the definition, any SOS tensor is positive semi-definite. On the other hand, the converse is not true, in general [14,15]. The importance of studying SOS tensors is that the problem for determining an even order symmetric tensor is an SOS tensor or not is equivalent to solving a semi-infinite linear programming problem, which can be done in polynomial time; while determining the positive semi-definiteness of a symmetric tensor is, in general, NP-hard. Interestingly, it was recently shown in [14] that for a so-called Z-tensor $\mathcal{A}$ where the off-diagonal elements are all non-positive, $\mathcal{A}$ is positive semi-definite if and only if it is a SOS tensor.

Tensor $\mathcal{A}$ is called a completely decomposable tensor if there are vectors $x_{j} \in \mathbb{R}^{n}, j \in[r]$ such that $\mathcal{A}$ can be written as sums of rank-one tensors generated by the vector $x_{j}$, that is,

$$
\mathcal{A}=\sum_{j \in[r]} x_{j}^{m} .
$$

If $x_{j} \in \mathbb{R}_{+}^{n}$ for all $j \in[r]$, then $\mathcal{A}$ is called a completely positive tensor [33]. It was shown that a strongly symmetric, hierarchically dominated nonnegative tensor is a completely positive tensor [33]. It can be directly verified that all even order completely positive tensors are SOS tensors, and so, are also positive semi-definite tensors. We note that verifying a tensor $\mathcal{A}$ is a completely decomposable or not, and finding its explicit rank one decomposition are highly nontrivial. This topic has attracted a lot of researchers and many important work has been established along this direction. For detailed discussions, see [6,16,33] and the reference therein.

We now characterize the SOS property and complete decomposability for even order generalized Cauchy tensors with nonzero entries.

Theorem 3.1. Let $\mathcal{C}$ be a generalized Cauchy tensor with even order $m$ and dimension $n$. Let $c=\left(c_{1}, \ldots, c_{n}\right)^{T} \in \mathbb{R}^{n}$ and $d=$ $\left(d_{1}, \ldots, d_{n}\right)^{T} \in \mathbb{R}^{n}$ be the generating vectors of $\mathcal{C}$. Assume $d_{i} \neq 0, i \in[n]$. Then, the following statements are equivalent:

(i) the generalized Cauchy tensor $\mathcal{C}$ is a completely decomposable tensor;

(ii) the generalized Cauchy tensor $\mathcal{C}$ is an SOS tensor;

(iii) the generalized Cauchy tensor $\mathcal{C}$ is positive semi-definite;

(iv) $c_{i}>0, i \in[n]$.

Proof. Since $m$ is even, by the definitions of completely decomposable tensor, SOS tensor and positive semi-definite tensor, we can easily obtain (i) $\Rightarrow$ (ii) and (ii) $\Rightarrow$ (iii).

[(iii) $\Rightarrow$ (iv)] Let $\mathcal{C}$ be an even order generalized Cauchy tensor which is positive semi-definite. Then

$$
\mathcal{C} e_{i}^{m}=\frac{d_{i}^{m}}{m c_{i}} \geq 0 .
$$

So $c_{i}>0$ for all $i \in[n]$.

$[(\mathrm{iv}) \Rightarrow(\mathrm{i})]$ Suppose that $c_{i}>0, i \in[n]$. Then, for any $x \in \mathbb{R}^{n}$,

$$
\begin{aligned}
f(x) & =\mathcal{C} x^{m}=\sum_{i_{1}, i_{2}, \ldots, i_{m}=1}^{n} \frac{d_{i_{1}} d_{i_{2}} \ldots d_{i_{m}}}{c_{i_{1}}+c_{i_{2}}+\cdots+c_{i_{m}}} x_{i_{1}} x_{i_{2}} \ldots x_{i_{m}} \\
& =\sum_{i_{1}, i_{2}, \ldots, i_{m}=1}^{n}\left(\int_{0}^{1} t^{c_{i_{1}}+c_{i_{2}}+\cdots+c_{i_{m}}-1} d_{i_{1}} d_{i_{2}} \ldots d_{i_{m}} x_{i_{1}} x_{i_{2}} \ldots x_{i_{m}} d t\right) \\
& =\int_{0}^{1}\left(\sum_{i_{1}, i_{2}, \ldots, i_{m}=1}^{n} t^{c_{i_{1}}+c_{i_{2}}+\cdots+c_{i_{m}}-1} d_{i_{1}} d_{i_{2}} \ldots d_{i_{m}} x_{i_{1}} x_{i_{2}} \ldots x_{i_{m}}\right) d t \\
& =\int_{0}^{1}\left(\sum_{i=1}^{n} t^{c_{i}-\frac{1}{m}} d_{i} x_{i}\right)^{m} d t .
\end{aligned}
$$

By the definition of Riemann integral, we have

$$
\mathcal{C} x^{m}=\lim _{k \rightarrow \infty} \sum_{j=1}^{k} \frac{\left(\sum_{i=1}^{n}\left(\frac{j}{k}\right)^{c_{i}-\frac{1}{m}} d_{i} x_{i}\right)^{m}}{k} .
$$


Let $\mathcal{C}_{k}$ be the symmetric tensor such that

$$
\begin{aligned}
\mathcal{C}_{k} x^{m} & =\sum_{j=1}^{k} \frac{\left(\sum_{i=1}^{n}\left(\frac{j}{k}\right)^{c_{i}-\frac{1}{m}} d_{i} x_{i}\right)^{m}}{k} \\
& =\sum_{j=1}^{k}\left(\sum_{i=1}^{n} \frac{\left(\frac{j}{k}\right)^{c_{i}-\frac{1}{m}} d_{i}}{k^{\frac{1}{m}}} x_{i}\right)^{m} \\
& =\sum_{j=1}^{k}\left(\left\langle u^{j}, x\right\rangle\right)^{m},
\end{aligned}
$$

where

$$
u^{j}=\left(\frac{\left(\frac{j}{k}\right)^{c_{1}-\frac{1}{m}} d_{1}}{k^{\frac{1}{m}}}, \ldots, \frac{\left(\frac{j}{k}\right)^{c_{n}-\frac{1}{m}} d_{n}}{k^{\frac{1}{m}}}\right) \in \mathbb{R}^{n}, j=1, \ldots, k .
$$

Let $\mathrm{CD}_{m, n}$ denote the set consisting of all completely decomposable tensor with order $m$ and dimension $n$. From [20, Theorem 1], $\mathrm{CD}_{m, n}$ is a closed convex cone when $m$ is even. It then follows that $\mathcal{C}=\lim _{k \rightarrow \infty} \mathcal{C}_{k}$ is also a completely decomposable tensor.

Next, we provide a sufficient and necessary condition for the complete positivity of a generalized Cauchy tensor with nonzero entries, in terms of its generating vectors.

Theorem 3.2. Let $\mathcal{C}$ be a generalized Cauchy tensor defined as in Theorem 3.1 with generating vectors $c=\left(c_{1}, \ldots, c_{n}\right)^{T} \in \mathbb{R}^{n}$ and $d=\left(d_{1}, \ldots, d_{n}\right)^{T} \in \mathbb{R}^{n}$. Assume $d_{i} \neq 0, i \in[n]$. Then $\mathcal{C}$ is a completely positive tensor if and only if $c_{i}>0$ and $d_{i}>0, i \in[n]$.

Proof. For necessary condition, suppose that $\mathcal{C}$ is a completely positive tensor. Then, for any vector $x \in \mathbb{R}_{+}^{n}$, we must have $\mathcal{C} x^{m} \geq 0$. So, $\mathcal{C} e_{i}^{m}=\frac{d_{i}^{m}}{m c_{i}} \geq 0$. This implies that $c_{i}>0, i \in[n]$. To finish the proof, we only need to show $d_{i}>0, i \in[n]$. To see this, we proceed by the method of contradiction and suppose that

$$
I_{-}:=\left\{i \in\{1, \ldots, n\}: d_{i}<0\right\} \neq \emptyset .
$$

Denote $r$ to be the cardinality of $I_{-}$. Without loss of generality, we assume that $I_{-}=\{1, \ldots, r\}$. Then, $d_{1}<0$ and $d_{r+1}>0$, and hence, the $(r+1,1, \ldots, 1)$ th entry of $\mathcal{C}$ satisfies

$$
\mathcal{C}_{r+11 \ldots 1}=\frac{d_{r+1} d_{1}^{m-1}}{c_{r+1}+(m-1) c_{1}}<0 .
$$

Note that each entry of a completely positive tensor must be a nonnegative number. This makes contradiction, and hence, the necessary condition follows.

To prove the sufficient condition, from (3.2) and (3.3), we know that

$$
\mathcal{C} x^{m}=\lim _{k \rightarrow \infty} \sum_{j=1}^{k}\left(\left\langle u^{j}, x\right\rangle\right)^{m} .
$$

As $c_{i}>0$ and $d_{i}>0, i \in[n]$, (3.4) implies that $u^{j} \in \mathbb{R}_{+}^{n}, j \in[k]$. So each $\mathcal{C}_{k}$ is a completely positive tensor. Let $\mathrm{CP}_{m, n}$ denote the set consisting of all completely positive tensors with order $m$ and dimension $n$. From [33], $\mathrm{CP}_{m, n}$ is a closed convex cone for any $m, n \in \mathbb{N}$. It then follows that $\mathcal{C}=\lim _{k \rightarrow \infty} \mathcal{C}_{k}$ is also a completely positive tensor.

Let $\mathcal{A}=\left(a_{i_{1} \ldots i_{m}}\right)$ and $\mathcal{B}=\left(b_{i_{1} \ldots i_{m}}\right)$ be two real tensors with order $m$ and dimension $n$. Then their Hadamard product is a real order $m$ dimension $n$ tensor

$$
\mathcal{A} \circ \mathcal{B}=\left(a_{i_{1} \ldots i_{m}} b_{i_{1} \ldots i_{m}}\right)
$$

From Proposition 1 of [33], we know that the Hadamard product of two completely positive tensors is also a completely positive tensor. So, we have the following conclusion.

Corollary 3.1. Let $\mathcal{C}_{1}$ and $\mathcal{C}_{2}$ be two positive semi-definite Cauchy tensors. Then the Hadamard product $\mathcal{C}_{1} \circ \mathcal{C}_{2}$ is also positive semidefinite.

Next, we have the following theorem on H-eigenvalues of nonnegative Cauchy tensors. By [40], we know that each nonnegative symmetric tensor has at least one H-eigenvalue, which is the largest modulus of its eigenvalues. Here, for nonnegative Cauchy tensors, all the $\mathrm{H}$-eigenvalues must be nonnegative.

Theorem 3.3. Let $\mathcal{C}$ be a nonnegative Cauchy tensor with order $m$ dimension $n$. Let $c=\left(c_{1}, c_{2}, \ldots, c_{n}\right)^{T}$ be the generating vector of tensor $\mathcal{C}$. Then all $\mathrm{H}$-eigenvalues of Cauchy tensor $\mathcal{C}$ are nonnegative. 
Proof. In the case where $m$ is even, since $\mathcal{C}$ is nonnegative and the definition of a Cauchy tensor, we have $c_{i}>0, i=1, \ldots, n$. From Theorem 2.1 of [3], we know that $\mathcal{C}$ is positive semi-definite. Then, Theorem 5 of [27] gives us that all H-eigenvalues of $\mathcal{C}$ are nonnegative.

We now consider the case where $m$ is odd. Let $\lambda$ be an arbitrary H-eigenvalue of $\mathcal{C}$ with an H-eigenvector $x \neq 0$. By the definition of $H$-eigenvalue, it holds that

$$
\begin{aligned}
\lambda x_{i}^{m-1} & =\left(\mathcal{C} x^{m-1}\right)_{i} \\
& =\sum_{i_{2}, \ldots, i_{m}=1}^{n} \frac{x_{i_{2}} x_{i_{3}} \ldots x_{i_{m}}}{c_{i}+c_{i_{2}}+\cdots+c_{i_{m}}} \\
& =\sum_{i_{2}, \ldots, i_{m}=1}^{n}\left(\int_{0}^{1} t^{c_{i}+c_{i_{2}}+\cdots+c_{i_{m}}-1} x_{i_{2}} x_{i_{3}} \ldots x_{i_{m}} d t\right) \\
& =\int_{0}^{1}\left(\sum_{i_{2}, \ldots, i_{m}=1}^{n} t^{c_{i}+c_{i_{2}}+\cdots+c_{i_{m}}-1} x_{i_{2}} x_{i_{3}} \ldots x_{i_{m}}\right) d t \\
& =\int_{0}^{1}\left(\sum_{j=1}^{n} t^{c_{j}+\frac{c_{i}-1}{m-1}} x_{j}\right)^{m-1} d t .
\end{aligned}
$$

This implies that $\lambda \geq 0$ since $m$ is odd. Thus, the desired result holds.

Now, we give an example to verify the result of Theorem 3.3. Here, we only show the nonnegativity of $H$-eigenvalues for an odd order nonnegative Cauchy tensor since all even order nonnegative Cauchy tensors are always positive semi-definite [3].

Example 3.1. Let $\mathcal{C}=\left(c_{i_{1} i_{2} i_{3}}\right)$ be a nonnegative Cauchy tensor with generating vector $c=(1,1,2)$. Then, it has entries such that

$$
\begin{aligned}
& c_{111}=c_{222}=\frac{1}{3}, c_{333}=\frac{1}{6}, c_{112}=c_{121}=c_{211}=\frac{1}{3}, c_{113}=c_{131}=c_{311}=\frac{1}{4}, \\
& c_{122}=c_{221}=c_{212}=\frac{1}{3}, c_{133}=c_{331}=c_{313}=\frac{1}{5}, c_{223}=c_{232}=c_{322}=\frac{1}{4}, \\
& c_{233}=c_{332}=c_{323}=\frac{1}{5}, c_{123}=c_{132}=c_{312}=c_{321}=c_{231}=c_{213}=\frac{1}{4} .
\end{aligned}
$$

By Definition 2.1, to get all H-eigenvalues of $\mathcal{C}$ is equivalent to solving the following system:

$$
\left\{\begin{array}{l}
\frac{1}{3} x_{1}^{2}+\frac{2}{3} x_{1} x_{2}+\frac{1}{2} x_{1} x_{3}+\frac{1}{3} x_{2}^{2}+\frac{1}{5} x_{3}^{2}+\frac{1}{2} x_{2} x_{3}=\lambda x_{1}^{2} \\
\frac{1}{3} x_{2}^{2}+\frac{2}{3} x_{1} x_{2}+\frac{1}{2} x_{2} x_{3}+\frac{1}{3} x_{1}^{2}+\frac{1}{5} x_{3}^{2}+\frac{1}{2} x_{1} x_{3}=\lambda x_{2}^{2} \\
\frac{1}{6} x_{3}^{2}+\frac{2}{5} x_{1} x_{3}+\frac{2}{5} x_{2} x_{3}+\frac{1}{4} x_{2}^{2}+\frac{1}{4} x_{1}^{2}+\frac{1}{2} x_{1} x_{2}=\lambda x_{3}^{2}
\end{array}\right.
$$

Since Cauchy tensor $\mathcal{C}$ is nonnegative, it always has H-eigenvalue i.e. the above system at least has a solution $\lambda \in \mathbb{R}$. Next, we will prove that $\lambda$ may not be negative. Without loss of generality, choose one equation from (3.5) such that

$$
\frac{1}{3} x_{1}^{2}+\frac{2}{3} x_{1} x_{2}+\frac{1}{2} x_{1} x_{3}+\frac{1}{3} x_{2}^{2}+\frac{1}{5} x_{3}^{2}+\frac{1}{2} x_{2} x_{3}=\lambda x_{1}^{2} .
$$

It is east to see that the left of the equality in (3.6) is quadratic form and the corresponded symmetric matrix is

$$
\left(\begin{array}{lll}
\frac{1}{3} & \frac{1}{3} & \frac{1}{4} \\
\frac{1}{3} & \frac{1}{3} & \frac{1}{4} \\
\frac{1}{4} & \frac{1}{4} & \frac{1}{5}
\end{array}\right)
$$

By direct computation, we obtain that the matrix is positive semi-definite, which implies that

$$
\frac{1}{3} x_{1}^{2}+\frac{2}{3} x_{1} x_{2}+\frac{1}{2} x_{1} x_{3}+\frac{1}{3} x_{2}^{2}+\frac{1}{5} x_{3}^{2}+\frac{1}{2} x_{2} x_{3} \geq 0, \forall x \in \mathbb{R}^{3} .
$$

Thus, all H-eigenvalues of Cauchy tensor $\mathcal{C}$ are nonnegative. 


\section{Further properties on Hankel tensors}

Hankel tensors arise from signal processing and some other applications [1,9,25,28]. Recall that an order $m$ dimension $n$ tensor $\mathcal{A}=\left(a_{i_{1} i_{2} \ldots i_{m}}\right)$ is called a Hankel tensor if there is a vector $v=\left(v_{0}, v_{1}, \ldots, v_{(n-1) m}\right)^{T}$ such that

$$
a_{i_{1} i_{2} \ldots i_{m}}=v_{i_{1}+i_{2}+\cdots+i_{m}-m}, \forall i_{1}, i_{2}, \ldots, i_{m} \in[n] .
$$

Such a vector $v$ is called the generating vector of Hankel tensor $\mathcal{A}$.

For any $k \in \mathbb{N}$, let $s(k, m, n)$ be the number of distinct sets of indices $\left(i_{1}, i_{2}, \ldots, i_{m}\right), i_{j} \in[n], j \in[m]$ such that $i_{1}+i_{2}+\cdots+i_{m}-$ $m=k$. For example, $s(0, m, n)=1, s(1, m, n)=m, s(2, m, n)=\frac{m(m+1)}{2}$. Suppose $\mathcal{P}=\left(p_{i_{1} i_{2} \ldots i_{(n-1) m}}\right)$ is an order $(n-1) m \operatorname{dimen-}$ sion 2 tensor defined by

$$
p_{i_{1} i_{2} \cdots i_{(n-1) m}}=\frac{s(k, m, n) v_{k}}{\left(\begin{array}{c}
(n-1) m \\
k
\end{array}\right)},
$$

where $k=i_{1}+i_{2}+\cdots+i_{(n-1) m}-(n-1) m$. Then tensor $\mathcal{P}$ is called the associated plane tensor of Hankel tensor $\mathcal{A}$. When $n=2$, it is obvious that $\mathcal{P}=\mathcal{A}$.

In [28], it was proved that, if a Hankel tensor is copositive, then its associated plane tensor $\mathcal{P}$ is copositive and the associated plane tensor is positive semi-definite if the Hankel tensor is positive semi-definite. Since the associated plane tensor $\mathcal{P}$ has dimension 2, we can use the Z-eigenvalue method in [30] to check its positive semi-definiteness (alternatively, noting that any 2-dimensional symmetric tensor is positive semi-definite if and only if it is a sums-of-squares tensor, we can also verify the positive semi-definiteness of the associated plane tensor by solving a semi-definite programming problem). Thus, the positive semi-definiteness of the associated plane tensor is a checkable necessary condition for the positive semi-definiteness of even order Hankel tensors (see more discussion in [28]). This naturally raises the following questions: Can these necessary conditions be also sufficient? If not, are there any concrete counter-examples?

We first present a result stating that the positive semi-definiteness of the associated plane tensor is equivalent to the Vandermonde positive semi-definiteness of the original Hankel tensor.

Theorem 4.1. Let $\mathcal{A}$ be a Hankel tensor defined as in (4.1) with an even order $m$. Then, the associated plane tensor $\mathcal{P}$ is positive semi-definite if and only if $\mathcal{A}$ is Vandermonde positive semi-definite.

Proof. For necessary condition, let $u=\left(1, \mu, \mu^{2}, \ldots, \mu^{n-1}\right)^{T} \in \mathbb{R}^{n}$ be an arbitrary Vandermonde vector. If $\mu=0$, then we have

$$
\mathcal{A} u^{m}=\sum_{i_{1}, i_{2}, \ldots, i_{m} \in[n]} a_{i_{1} i_{2} \ldots i_{m}} u_{i_{1}} u_{i_{2}} \ldots u_{i_{m}}=v_{0} .
$$

By our assumption, for $y=(1,0)^{T} \in \mathbb{R}^{2}$, it follows that

$$
\mathcal{P} y^{(n-1) m}=\sum_{i_{1}, i_{2}, \ldots, i_{(n-1) m} \in[2]} p_{i_{1} i_{2} \ldots i_{(n-1) m}} y_{i_{1}} y_{i_{2}} \ldots y_{i_{(n-1) m}}=v_{0} \geq 0 .
$$

Combining this with (4.2), we obtain

$$
\mathcal{A} u^{m} \geq 0 .
$$

If $\mu \neq 0$, there exist $y_{1}, y_{2} \in \mathbb{R} \backslash\{0\}$ such that $\mu=\frac{y_{2}}{y_{1}}$. Let $y=\left(y_{1}, y_{2}\right)^{T} \in \mathbb{R}^{2}$. Then, we have

$$
\begin{aligned}
\mathcal{P} y^{(n-1) m} & =\sum_{i_{1}, i_{2}, \ldots, i_{(n-1) m} \in[2]} p_{i_{1} i_{2} \ldots i_{(n-1) m}} y_{i_{1}} y_{i_{2}} \ldots y_{i_{(n-1) m}} \\
& =y_{1}^{(n-1) m} \sum_{k=0}^{(n-1) m}\left(\begin{array}{c}
(n-1) m \\
k
\end{array}\right) \frac{s(k, m, n) v_{k}}{\left(\begin{array}{c}
(n-1) m \\
k
\end{array}\right)} \mu^{k} \\
& =y_{1}^{(n-1) m} \mathcal{A} u^{m} \\
& \geq 0 .
\end{aligned}
$$

By (4.3) and the fact that $m$ is even, for all Vandermonde vectors $u \in \mathbb{R}^{n}$, it follows that

$$
\mathcal{A} u^{m} \geq 0
$$

which implies Hankel tensor $\mathcal{A}$ is Vandermonde positive semi-definite.

For sufficiency, let $y=\left(y_{1}, y_{2}\right)^{T} \in \mathbb{R}^{2}$. We now verify that $\mathcal{P} y^{(n-1) m} \geq 0$. To see this, we first consider the case where $y_{1} \neq 0$. In this case, let $u=\left(1, \mu, \mu^{2}, \ldots, \mu^{n-1}\right)^{T} \in \mathbb{R}^{n}$, where $\mu=\frac{y_{2}}{y_{1}}$. From the analysis above, we have

$$
\mathcal{P} y^{(n-1) m}=y_{1}^{(n-1) m} \mathcal{A} u^{m} \geq 0
$$

since $m$ is even and $\mathcal{A}$ is Vandermonde positive semi-definite. 
On the other hand, if $y=\left(y_{1}, y_{2}\right)^{T} \in \mathbb{R}^{2}$ with $y_{1}=0$, then we let $y_{\epsilon}=\left(\epsilon, y_{2}\right)^{T} \in \mathbb{R}^{2}$ and $u=\left(1, \mu, \mu^{2}, \ldots, \mu^{n-1}\right)^{T} \in \mathbb{R}^{n}$, where $\mu=\frac{y_{2}}{\epsilon}, \epsilon>0$. By (4.4), we have

$$
\mathcal{P} y_{\epsilon}^{(n-1) m}=\epsilon^{(n-1) m} \mathcal{A} u^{m} \geq 0 .
$$

Combining this with the fact that $\epsilon \mapsto \mathcal{P} y_{\epsilon}^{(n-1) m}$ is a continuous mapping, it follows that

$$
\mathcal{P} y^{(n-1) m}=\lim _{\epsilon \rightarrow 0} \mathcal{P} y_{\epsilon}^{(n-1) m} \geq 0 .
$$

This then implies that plane tensor $\mathcal{P}$ is positive semi-definite and the desired result holds.

Below, we provide an example illustrating that a Hankel tensor which is Vandermonde positive semi-definite need not to be positive semi-definite. This example together with Theorem 4.1, also implies that the positive semi-definiteness of the associate plane tensor is not sufficient for positive semi-definiteness of the Hankel tensor.

Example 4.1. Let $\mathcal{A}$ be a Hankel tensor with order $m=4$ and dimension $n=3$. Let the generating vector of $\mathcal{A}$ be $v_{0}=1, v_{1}=$ $-1, v_{2}=1$ and $v_{3}=v_{4}=\cdots=v_{8}=0$. So, for any $u=\left(1, \mu, \mu^{2}\right)^{T} \in \mathbb{R}^{3}$,

$$
\begin{aligned}
\mathcal{A} u^{4} & =\sum_{i_{1}, i_{2}, i_{3}, i_{4} \in[3]} a_{i_{1} i_{2} i_{3} i_{4}} u_{i_{1}} u_{i_{2}} u_{i_{3}} u_{i_{4}} \\
& =\sum_{k=0}^{k=8} s(k, 4,3) v_{k} \mu^{k} \\
& =v_{0}+4 v_{1} \mu+10 v_{2} \mu^{2} \\
& =1-4 \mu+10 \mu^{2} \geq 0
\end{aligned}
$$

for all $\mu \in \mathbb{R}$. By Theorem 4.1, we know that the associated plane tensor $\mathcal{P}$ is positive semi-definite. We now verify that $\mathcal{A}$ is not positive semi-definite. To see this, let $x=(1,1,-1)^{T}$, then,

$$
\begin{aligned}
\mathcal{A} x^{4} & =\sum_{i_{1}, i_{2}, i_{3}, i_{4} \in[3]} v_{i_{1}+i_{2}+i_{3}+i_{4}-4} x_{i_{1}} x_{i_{2}} x_{i_{3}} x_{i_{4}} \\
& =v_{0} x_{1}^{4}+4 v_{1} x_{1}^{3} x_{2}+v_{2}\left(6 x_{2}^{2} x_{1}^{2}+4 x_{1}^{3} x_{3}\right) \\
& =x_{1}^{4}-4 x_{1}^{3} x_{2}+\left(6 x_{2}^{2} x_{1}^{2}+4 x_{1}^{3} x_{3}\right) \\
& =1-4+6-4=-1<0,
\end{aligned}
$$

which implies that Hankel tensor $\mathcal{A}$ is not positive semi-definite.

The following example shows that the the copositivity of the associated plane tensor is also not sufficient for the copositivity of the Hankel tensor, in general.

Example 4.2. Let $\mathcal{A}$ be a Hankel tensor with order $m=4$ and dimension $n=3$. Let the generating vector of $\mathcal{A}$ be $v_{0}=1, v_{1}=$ $-1, v_{2}=\frac{1}{2}, v_{3}=v_{4}=\cdots=v_{8}=0$. Let $x=\left(1, \frac{1}{2}, 0\right)^{T}$. Then, we have

$$
\mathcal{A} x^{4}=-\frac{1}{4}<0
$$

which implies that Hankel tensor $\mathcal{A}$ is not copositive. On the other hand, it holds that

$$
\mathcal{A} u^{4}=1-4 \mu+5 \mu^{2} \geq 0
$$

for any Vandermonde vector $u=\left(1, \mu, \mu^{2}\right)^{T} \in \mathbb{R}^{3}$. By Theorem 4.1, the associated plane tensor $\mathcal{P}$ is positive semi-definite. Thus, $\mathcal{P}$ is copositive.

A special class of Hankel tensor is the complete Hankel tensors. To recall the definition of a complete Hankel tensor, we note that, for a Hankel tensor $\mathcal{A}$ with order $m$ dimension $n$, if

$$
\mathcal{A}=\sum_{k=1}^{r} \alpha_{k}\left(u_{k}\right)^{m},
$$

where $\alpha_{k} \in \mathbb{R}, \alpha_{k} \neq 0, u_{k}=\left(1, \mu_{k}, \mu_{k}^{2}, \ldots, \mu_{k}^{n-1}\right)^{T} \in \mathbb{R}^{n}, k=1,2, \ldots, r$, for some $\mu_{i} \neq \mu_{j}$ for $i \neq j$, then, we say $\mathcal{A}$ has a Vandermonde decomposition. The corresponding vector $u_{k}, k=1, \ldots, r$ are called Vandermonde vectors and the minimum value of $r$ is called Vandermonde rank of $\mathcal{A}$ [28]. From Theorem 3 of [28], we know that $\mathcal{A}$ is a Hankel tensor if and only if it has a Vandermonde decomposition (4.5). If $\alpha_{k}>0$ for $k \in[r]$ in (4.5), then $\mathcal{A}$ is called a complete Hankel tensor.

In [28], it is proved that an even order complete Hankel tensor is positive semi-definite. Moreover, examples were also presented to show that the converse is, in general, not true. Here, in the following theorem, we show that if the Vandermonde rank of a Hankel tensor $\mathcal{A}$ is less than the dimension of the underlying space, then positive semi-definiteness of $\mathcal{A}$ is equivalent to the fact that $\mathcal{A}$ is a complete Hankel tensor, and so, is further equivalent to the SOS property of $\mathcal{A}$. 
Theorem 4.2. Let $\mathcal{A}$ be a Hankel tensor with an even order $m$. Assume that the Hankel tensor $\mathcal{A}$ has Vandermonde decomposition (4.5) with the Vandermonde rank $r$ satisfies $r \leq n$. Then, the following statements are equivalent:

(i) $\mathcal{A}$ is a positive semi-definite tensor;

(ii) $\mathcal{A}$ is a complete Hankel tensor.

(iii) $\mathcal{A}$ is an SOS tensor;

Proof. We first note that the implications $[($ ii $)] \Rightarrow[($ iii $)]$ and $[($ iii $)] \Rightarrow[(\mathrm{i})]$ are direct consequences from the definitions. Thus, to see the conclusion, we only need to prove $[(\mathrm{i})] \Rightarrow[(\mathrm{ii})]$. To do this, we proceed by the method of contradiction and assume that there exists at least one coefficient $\alpha_{i}$ in (4.5) which is negative. Without loss of generality, we assume that $\alpha_{1}<0$. For any $x=\left(x_{1}, x_{2}, \ldots, x_{n}\right)^{T} \in \mathbb{R}^{n}$, then we have

$$
\begin{aligned}
\mathcal{A} x^{m} & =\sum_{k=1}^{r} \alpha_{k}\left(u_{k}^{T} x\right)^{m} \\
& =\alpha_{1}\left(u_{1}^{T} x\right)^{m}+\alpha_{2}\left(u_{2}^{T} x\right)^{m}+\cdots+\alpha_{r}\left(u_{r}^{T} x\right)^{m} .
\end{aligned}
$$

Consider the following two homogeneous linear equation systems

$$
A x=\mathbf{0}, B x=\mathbf{0},
$$

where

$$
A=\left(\begin{array}{ccccc}
1 & \mu_{1} & \mu_{1}^{2} & \cdots & \mu_{1}^{n-1} \\
1 & \mu_{2} & \mu_{2}^{2} & \cdots & \mu_{2}^{n-1} \\
\vdots & \vdots & \vdots & \vdots & \vdots \\
1 & \mu_{r} & \mu_{r}^{2} & \cdots & \mu_{r}^{n-1}
\end{array}\right), \quad B=\left(\begin{array}{ccccc}
1 & \mu_{2} & \mu_{2}^{2} & \cdots & \mu_{2}^{n-1} \\
1 & \mu_{3} & \mu_{3}^{2} & \cdots & \mu_{3}^{n-1} \\
\vdots & \vdots & \vdots & \vdots & \vdots \\
1 & \mu_{r} & \mu_{r}^{2} & \cdots & \mu_{r}^{n-1}
\end{array}\right) .
$$

By conditions $r \leq n$, it is easy to get

$$
\operatorname{Rank}(A)=r \leq n, \operatorname{Rank}(B)=r-1<n,
$$

which imply that there is vector $x_{0} \in \mathbb{R}^{n}, x_{0} \neq \mathbf{0}$ such that

$$
A x_{0} \neq \mathbf{0}, B x_{0}=\mathbf{0} \text {. }
$$

Here, $\operatorname{Rank}(A)$ denotes the rank of matrix $A$. So, it holds that

$$
u_{1}^{T} x_{0} \neq 0, u_{i}^{T} x_{0}=0, i \in\{2,3, \ldots, r\} .
$$

Combining this with (4.6), we have

$$
\mathcal{A} x_{0}^{m}=\alpha_{1}\left(u_{1}^{T} x_{0}\right)^{m}<0
$$

since $m$ is even. However, this contradicts to the fact that $\mathcal{A}$ is positive semi-definite. Thus, all coefficients in (4.5) are positive and $\mathcal{A}$ is a complete Hankel tensor.

An interesting consequence of Theorem 4.2 is as follows: a necessary condition for a PNS (positive semi-definite but not sumof-squares) Hankel tensor $\mathcal{A}$ is that the Vandermonde rank $r$ of the Hankel tensor $\mathcal{A}$ is strictly larger than the dimension $n$ of the underlying space. We note that searching for a PNS Hankel tensor is a non-trivial task and is related to Hilbert's 17th question. Recently, some extensive study for PNS Hankel tensor has been initialed in [21].

Next, we provide some necessary conditions for the positive semi-definiteness of a Hankel tensor $\mathcal{A}$ in terms of the sign properties of the coefficients of its Vandermonde decomposition.

Proposition 4.1. Let $\mathcal{A}$ be a Hankel tensor with the Vandermonde decomposition (4.5). Suppose that $\mathcal{A}$ is positive semi-definite. Then,

(i) the coefficients of the Vandermonde decomposition satisfy

$$
\alpha_{1}+\alpha_{2}+\cdots+\alpha_{r} \geq 0
$$

(ii) if $r>n$, then the total number of positive coefficients of the Vandermonde decomposition is greater than or equal to $n$;

(iii) if $r \leq n$, then all coefficients of the Vandermonde decomposition are positive.

\section{Proof.}

(i) Since $\mathcal{A}$ is positive semi-definite, so we have

$$
\mathcal{A} e_{1}^{m}=\sum_{i=1}^{r} \alpha_{i}\left(u_{i}^{T} e_{1}\right)^{m}=\alpha_{1}+\alpha_{2}+\cdots+\alpha_{r} \geq 0 .
$$


(ii) Denote the total number of positive coefficients in (4.5) by $t$. Without loss of generality, let

$$
\alpha_{i}>0, i \in[t] ; \alpha_{j}<0, j \in\{t+1, t+2, \ldots, r\} .
$$

We proceed by the method of contradiction and suppose that $t<n$. If $t=0$, we can easily get a contradiction because $\mathcal{A}$ is positive semi-definite. If $0<t<n$, consider the following two linear equation systems

$$
A x=\mathbf{0}
$$

and

$$
B x=\mathbf{0},
$$

where

$$
A=\left(\begin{array}{ccccc}
1 & \mu_{1} & \mu_{1}^{2} & \cdots & \mu_{1}^{n-1} \\
1 & \mu_{2} & \mu_{2}^{2} & \cdots & \mu_{2}^{n-1} \\
\vdots & \vdots & \vdots & \vdots & \vdots \\
1 & \mu_{t} & \mu_{t}^{2} & \cdots & \mu_{t}^{n-1}
\end{array}\right), \quad B=\left(\begin{array}{ccccc}
1 & \mu_{1} & \mu_{1}^{2} & \cdots & \mu_{1}^{n-1} \\
1 & \mu_{2} & \mu_{2}^{2} & \cdots & \mu_{2}^{n-1} \\
\vdots & \vdots & \vdots & \vdots & \vdots \\
1 & \mu_{r} & \mu_{r}^{2} & \cdots & \mu_{r}^{n-1}
\end{array}\right) .
$$

Noting that $\operatorname{Rank}(A)=t<n$ and $\operatorname{Rank}(B)=n$, basic linear algebra theory implies that the system (4.7) has nonzero solutions and system (4.8) has only zero solution. Thus, there exists $\bar{x} \in \mathbb{R}^{n}, \bar{x} \neq 0$ such that

$$
u_{i}^{T} \bar{x}=0, i \in[t] \text { and }\left(u_{t+1}^{T} \bar{x}, \ldots, u_{r}^{T} \bar{x}\right)^{T} \neq \mathbf{0} .
$$

Note that the order $m$ is an even number (as $\mathcal{A}$ is positive semi-definite). This implies that

$$
\mathcal{A} \bar{x}^{m}=\sum_{j=t+1}^{r} \alpha_{j}\left(u_{j}^{T} \bar{x}\right)^{m}<0 .
$$

This contradicts with the fact that $\mathcal{A}$ is positive semi-definite. Then we get $t \geq n$.

(iii) If $r \leq n$, then the conclusion is a direct result of Theorem 4.2.

The following example shows that $r \leq n$ in Theorem 4.2 is necessary and the results (i), (ii) of Proposition 4.1 are not sufficient.

Example 4.3. Let $\mathcal{A}$ be 4 th order 2 dimension Hankel tensor with Vandermonde decomposition such that

$\mathcal{A}=\left(a_{i_{1} i_{2} i_{3} i_{4}}\right)=x^{4}+y^{4}-z^{4}$,

where $x=(1,0), y=(1,1), z=(1,-1)$ are Vandermonde vectors in $\mathbb{R}^{2}$. We first prove that the Vandermonde rank of $\mathcal{A}$ is $r=3$.

(I) Suppose $\mathcal{A}=\alpha u^{4}, \alpha \in \mathbb{R}, \alpha \neq 0, u=(1, \mu) \in \mathbb{R}^{2}$. Then, by (4.9), we obtain

$$
a_{1111}=\alpha=1, a_{1112}=\alpha \mu=2, a_{1122}=\alpha \mu^{2}=0,
$$

which are contradictive equations. So, the Vandermonde rank of $\mathcal{A}$ satisfies $r \geq 2$.

(II) Suppose $\mathcal{A}=\alpha_{1} u_{1}^{4}+\alpha_{2} u_{2}^{4}$, where $u_{1}=\left(1, \mu_{1}\right), u_{2}=\left(1, \mu_{2}\right) \in \mathbb{R}^{2}, \mu_{1} \neq \mu_{2}$, and $\alpha_{1}, \alpha_{2}$ are nonzero real numbers. Then, by (4.9), we have the following system

$$
\left\{\begin{array}{l}
\alpha_{1}+\alpha_{2}=1 \\
\alpha_{1} \mu_{1}+\alpha_{2} \mu_{2}=2 \\
\alpha_{1} \mu_{1}^{2}+\alpha_{2} \mu_{2}^{2}=0 \\
\alpha_{1} \mu_{1}^{3}+\alpha_{2} \mu_{2}^{3}=2 \\
\alpha_{1} \mu_{1}^{4}+\alpha_{2} \mu_{2}^{4}=0
\end{array}\right.
$$

We first prove that $\mu_{1} \neq 1, \mu_{2} \neq 1$. By contradiction, if $\mu_{1}=1$, then by (2) (4), we have

$$
\alpha_{2} \mu_{2}\left(\mu_{2}^{2}-1\right)=0 \text {, }
$$

which implies that $\mu_{2}=0$ or $\mu_{2}=-1\left(\mu_{2}\right.$ can not be 1 since $\left.\mu_{1} \neq \mu_{2}\right)$. If $\mu_{1}=1, \mu_{2}=0$, we get a contradiction from (2) and (3); if $\mu_{1}=1, \mu_{2}=-1$, we get another contradiction from (1) and (3). Thus, $\mu_{1} \neq 1$. Similarly, we can prove that $\mu_{2} \neq 1$.

On the other hand, by (2)-(5), it holds that

$$
\alpha_{1} \mu_{1}\left(\mu_{1}^{2}-1\right)=\alpha_{2} \mu_{2}\left(1-\mu_{2}^{2}\right)
$$

and

$$
\alpha_{1} \mu_{1}^{2}\left(\mu_{1}^{2}-1\right)=\alpha_{2} \mu_{2}^{2}\left(1-\mu_{2}^{2}\right)
$$


By (4.10), (4.11), it follows that $\mu_{1}=\mu_{2}$, which is a contradiction. Thus, the Vandermonde rank of the Hankel tensor $\mathcal{A}$ is $r=3$.

For this Hankel tensor $\mathcal{A}$, it is easy to check that conditions (i), (ii) in Proposition 4.1 hold. But $\mathcal{A}$ is not positive semi-definite since that

$$
\mathcal{A} x_{0}^{4}=-15<0, x_{0}=(1,-1) \in \mathbb{R}^{2} .
$$

\section{Properties of Cauchy-Hankel tensors}

In the literature, there is an important class of structured matrices called Cauchy-Hankel matrices which is closely related with Cauchy matrices and Hankel matrices simultaneously $[11,35,36]$. A matrix $A$ is called a Cauchy-Hankel matrices if it can be formulated as

$$
A=\left(\frac{1}{g+h(i+j)}\right)_{i, j=1}^{n},
$$

where $g$ and $h$ are real constants such that $h \neq 0$ and $\frac{g}{h}$ is not an integer [2].

As a natural extension of Cauchy-Hankel matrix, a tensor $\mathcal{A}=\left(a_{i_{1} i_{2} \ldots i_{m}}\right)$ with order $m$ and dimension $n$ is called a CauchyHankel tensor if

$$
a_{i_{1} i_{2} \ldots i_{m}}=\frac{1}{g+h\left(i_{1}+i_{2}+\cdots+i_{m}\right)}, i_{j} \in[n], j \in[m],
$$

where $g, h \neq 0 \in \mathbb{R}$ and $\frac{g}{h}$ is not an integer.

It is obvious that a Cauchy-Hankel tensor is a symmetric tensor. From Definition 3.1, we know that a Cauchy-Hankel tensor defined by (5.1) is a Cauchy tensor [3] with generating vector

$$
c=\left(\frac{g}{m}+h, \frac{g}{m}+2 h, \ldots, \frac{g}{m}+n h\right)^{T} \in \mathbb{R}^{n},
$$

and it is a Hankel tensor $[25,28]$ at the same time with

$$
v_{k}=\frac{1}{g+h(k+m)}, k \in\{0,1,2, \ldots,(n-1) m\} .
$$

Theorem 5.1. Let $\mathcal{A}$ be a Cauchy-Hankel tensor defined as in (5.1) with even order $m$. Then, $\mathcal{A}$ is positive definite if and only if

$$
g+m h>0, g+n m h>0 .
$$

Proof. For necessary condition, since $\mathcal{A}$ is positive definite, so we have

$$
\mathcal{A} e_{1}^{m}=\frac{1}{g+m h}>0, \mathcal{A} e_{n}^{m}=\frac{1}{g+m n h}>0,
$$

and the desired results hold.

For sufficiency, since

$$
g+m h>0, g+n m h>0,
$$

it follows that

$$
g+s m h>0, \forall s \in\{1,2, \ldots, n\} .
$$

Combining Theorem 2.3 of [3] and the fact that

$$
g+i m h \neq g+j m h, \forall i, j \in[n], i \neq j,
$$

we know that $\mathcal{A}$ is positive definite and the desired result follows.

Next, we define the homogeneous polynomial $f(x)$ as below

$$
f(x)=\mathcal{A} x^{m}=\sum_{i_{1}, i_{2}, \ldots, i_{m} \in[n]} a_{i_{1} i_{2} \ldots i_{m}} x_{i_{1}} x_{i_{2}} \ldots x_{i_{m}},
$$

for $x=\left(x_{1}, x_{2}, \ldots, x_{n}\right)^{T} \in \mathbb{R}^{n}$. Let $x, y \in X \subseteq \mathbb{R}^{n}$. If $f(x) \geq f(y)$ for any $x \geq y(x \leq y$ respectively), then we say $f(x)$ is monotonically increasing (monotonically decreasing respectively) in $X$. If $f(x)>f(y)$ for any $x \geq y, x \neq y(x \leq y, x \neq y$ respectively), then we say $f(x)$ is strict monotonically increasing (strict monotonically decreasing respectively) in $X$.

When $\mathcal{A}$ is a Cauchy tensor with even order, it has been proved that $f(x)$ is strict monotonically increasing in $\mathbb{R}_{+}^{n}$ if the Cauchy tensor $\mathcal{A}$ is positive definite; while the converse need not to be true [3]. For even order Cauchy-Hankel tensors, we have the following conclusion, which is stronger than the corresponded conclusion listed in [3]. 
Theorem 5.2. Let $\mathcal{A}$ be a Cauchy-Hankel tensor defined as in (5.1) with an even order $m$. Then, $\mathcal{A}$ is positive definite if and only if $f(x)=\mathcal{A} x^{m}$ is strict monotonically increasing in $\mathbb{R}_{+}^{n}$.

Proof. For the only if part, suppose $x, y \in \mathbb{R}_{+}^{n}, x \geq y$ and $x \neq y$, which means that there exists at least one subscript $i$ satisfying $x_{i}$ $>y_{i}$. Then, we have

$$
\begin{aligned}
f(x)-f(y) & =\mathcal{A} x^{m}-\mathcal{A} y^{m} \\
& =\sum_{i_{1}, i_{2}, \ldots, i_{m} \in[n]} \frac{x_{i_{1}} x_{i_{2}} \ldots x_{i_{m}}-y_{i_{1}} y_{i_{2}} \ldots y_{i_{m}}}{g+h\left(i_{1}+i_{2}+\cdots+i_{m}\right)} \\
& =\frac{x_{i}^{m}-y_{i}^{m}}{g+i m h}+\sum_{i_{1} i_{2} \ldots i_{m} \neq i i \ldots i} \frac{x_{i_{1}} x_{i_{2}} \ldots x_{i_{m}}-y_{i_{1}} y_{i_{2}} \ldots y_{i_{m}}}{g+h\left(i_{1}+i_{2}+\cdots+i_{m}\right)} .
\end{aligned}
$$

Since $\mathcal{A}$ is positive definite, by Theorem 5.1, we obtain

$$
g+k m h>0, \forall k \in[n] .
$$

So, we obtain

$$
\frac{x_{i}^{m}-y_{i}^{m}}{g+i m h}>0
$$

and

$$
\sum_{i_{1} i_{2} \ldots i_{m} \neq i i \ldots i} \frac{x_{i_{1}} x_{i_{2}} \ldots x_{i_{m}}-y_{i_{1}} y_{i_{2}} \ldots y_{i_{m}}}{g+h\left(i_{1}+i_{2}+\cdots+i_{m}\right)} \geq 0
$$

Thus, we have

$$
f(x)-f(y)>0,
$$

which implies that $f(x)$ is strict monotonically increasing in $\mathbb{R}_{+}^{n}$.

For the if part, note that $e_{i} \in \mathbb{R}_{+}^{n}$ and $e_{i} \geq \mathbf{0}, e_{i} \neq \mathbf{0}, i=1, n$. It then follows that

$$
f\left(e_{1}\right)-f(\mathbf{0})=\mathcal{A} e_{1}^{m}=\frac{1}{g+m h}>0
$$

and

$$
f\left(e_{n}\right)-f(\mathbf{0})=\mathcal{A} e_{n}^{m}=\frac{1}{g+n m h}>0 .
$$

By Theorem 5.1, we know that Cauchy-Hankel tensor $\mathcal{A}$ is positive definite and the desired results hold.

Theorem 5.1 and Theorem 5.2 provide a convenient checkable condition to verify the positive definiteness of the CauchyHankel tensor, and the strict monotonicity of the multivariate polynomial corresponding to the tensor. Here, we present several examples to show the efficiency of the theory conclusions.

Example 5.1. Suppose $\mathcal{A}=\left(a_{i_{1} i_{2} i_{3} i_{4}}\right)$ is a Cauchy-Hankel tensor such that

$$
a_{i_{1} i_{2} i_{3} i_{4}}=\frac{1}{9-2\left(i_{1}+i_{2}+i_{3}+i_{4}\right)}, i_{j} \in[3], j \in[4] .
$$

Here, it takes $g=9, h=-2$ and $m=4, n=3$. Since $g+m h>0, g+m n h<0$, tensor $\mathcal{A}$ is not positive definite and strict monotonically increasing in $\mathbb{R}_{+}^{n}$. In fact, it holds that

$$
\mathcal{A} e_{2}^{4}=-\frac{1}{7}, \mathcal{A} e_{2}^{4}<\mathcal{A} \mathbf{0}^{4}
$$

Example 5.2. Let $\mathcal{A}=\left(a_{i_{1} i_{2} i_{3} i_{4} i_{5} i_{6}}\right)$ be a tensor such that

$$
a_{i_{1} i_{2} i_{3} i_{4}}=\frac{1}{100-3\left(i_{1}+i_{2}+i_{3}+i_{4}+i_{5}+i_{6}\right)}, i_{j} \in[4], j \in[6] .
$$

By Theorem 5.1, Theorem 5.2 and the fact that

$$
g+m h=100-6 \cdot 3=72>0, g+m n h=100-6 \cdot 4 \cdot 3=18>0,
$$

$\mathcal{A}$ is positive definite and $\mathcal{A} x^{m}$ is strict monotonically increasing. 


\section{Final remarks}

In this article, we present various new results on Cauchy tensors and Hankel tensors which complements the existing literature. Firstly, we show that generalized positive semi-definite Cauchy tensors with nonzero entries are SOS tensors. Furthermore, sufficient and necessary conditions are given to guarantee an even order generalized Cauchy tensor is a completely positive tensor. The nonnegativity of H-eigenvalues of nonnegative Cauchy tensors are also established. For Hankel tensors, we prove that it is Vandermonde positive semi-definite if and only if the associated plane tensor is positive semi-definite. We also show that, if the Vandermonde rank of a Hankel tensor $\mathcal{A}$ is less than the dimension of the underlying space, then positive semi-definiteness of $\mathcal{A}$ is equivalent to the fact that $\mathcal{A}$ is a complete Hankel tensor, and so, is further equivalent to the SOS property of $\mathcal{A}$. Finally, properties of Cauchy-Hankel tensors are also given.

\section{Acknowledgments}

The authors are thankful to the two anonymous referees for their meaningful suggestions to improve the paper.

\section{References}

[1] R. Badeau, R. Boyer, Fast multilinear singular value decomposition for structured tensors, SIAM J. Matrix Anal. Appl. 30 (2008) 1008-1021.

[2] D. Bozkurt, On the norms of Hadamard product of Cauchy-Toeplitz and Cauchy-Hankel matrices, Linear Multilinear Algebra 44 (1998) 341-346.

[3] H. Chen, L. Qi, Positive Definiteness and Semi-Definiteness of Even Order Symmetric Cauchy Tensors, J. Ind. Manag. Optim. 11 (2015) $1263-1274$.

[4] Z. Chen, L. Qi, Circulant Tensors with Applications to Spectral Hypergraph Theory and Stochastic Process, to appear in: Journal of Industrial and Management Optimization (2014) arXiv:1312.2752v7.

[5] K.C. Chang, K. Pearson, T. Zhang, On eigenvalue problems of real symmetric tensors, J. Math. Anal. Appl. 350 (2009) 416-422.

[6] P. Comon, G. Golub, L.-H. Lim, B. Mourrain, Symmetric tensors and symmetric tensor rank, SIAM J. Matrix Anal. Appl. 30 (3) (2008) $1254-1279$.

[7] J. Cooper, A. Dutle, Spectral of Hypergraphs, Linear Algebra Appl. 436 (9) (2012) 3268-3292.

[8] W. Ding, L. Qi, Y. Wei, M-Tensors and Nonsingular M-Tensors, Linear Algebra Appl. 439 (2013) 3264-3278.

[9] W. Ding, L. Qi, Y. Wei, Fast Hankel Tensor-Vector Products and Application to Exponential Data Fitting, Numer. Linear Algebra Appl. 22 (2015) 814-832.

[10] M. Fiedler, Notes on Hilbert and Cauchy matrices, Linear Algebra Appl. 432 (2010) 351-356.

[11] A.D. Güngör, Lower bounds for the norms of Cauchy-Toeplitz and Cauchy-Hankel matrices, Appl. Math. Comput. 157 (3) (2004) 599-604.

[12] J. He, T.Z. Huang, Inequalities for M-tensors, J. Inequ. Appl. (2014) 114.

[13] C.J. Hillar, L.H. Lim, Most tensor problems are NP-hard, J. ACM 60 (6) (2013) 45

[14] S. Hu, G. Li, L. Qi, A tensor analogy of Yuan's alternative theorem and polynomial optimization with sign structure, J. Optim. Theory Appl., arXiv:1407.2348.

[15] S. Hu, G. Li, L. Qi, Y. Song, Finding the maximum eigenvalue of essentially nonnegative symmetric tensors via sum of squares programming, J. Optim. Theory Appl. 158 (2013) 717-738.

[16] T.G. Kolda, B.W. Bader, Tensor decompositions and applications, SIAM Rev. 51 (3) (2009) 455-500.

[17] L. De Lathauwer, B. De Moor, From matrix to tensor: Multilinear algebra and signal processing, in: J. McWhirter (Ed.), Mathematics in Signal Processing IV, Selected papers presented at 4th IMA Int. Conf. on Mathematics in Signal Processing, Oxford University Press, Oxford, United Kingdom, 1998, pp. 1-15.

[18] J.B. Lasserre, Global optimization with polynomials and the problem of moments, SIAM J. Optim. 11 (2011) $796-817$.

[19] M. Laurent, Sum of squares, moment matrices and optimization over polynomials, in: M. PUtinar, S. Sullivant (Eds.), Emerging Applications of Algebra Geometry, Vol. 149 of IMA Volumes in Mathematics and its Applications, Springer, 2009, pp. 157-270.

[20] G. Li, L. Qi, Y. Xu, SOS-Hankel Tensors: Theory and Application, 2014, arXiv:1410.6989. in press.

[21] G. Li, L. Qi, Q. Wang, Are there sixth order three dimensional PNS Hankel tensors? November 2014, arXiv:1411.2368.

[22] G. Li, L. Qi, G. Yu, Semismoothness of the maximum eigenvalue function of a symmetric tensor and its application, Linear Algebra Appl. 438 (2013) 813-833.

[23] G. Li, L. Qi, G. Yu, The Z-eigenvalues of a asymmetric tensor and its application to spectral hypergraph theory, Numerical Linear Algebra Appl. 20 (2013) 1001-1029.

[24] L.H. Lim, Singular values and eigenvalues of tensors, a variational approach, in: Proceedings of the 1st IEEE International workshop on computational advances of multi-tensor adaptive processing, 2005, pp. 129-132.

[25] J.M. Papy, L. De Lauauwer, S.V. Huffel, Exponential data fitting using multilinear algebra: The single-channel and multi-channel case, Numer. Linear Algebra Appl. 12 (2005) 809-826.

[26] G. Pólya, G. Szegö, Zweiter Band, Springer, Berlin, 1925

[27] L. Qi, Eigenvalue of a real super symmetric tensor, J. Symbol. Comput. 40 (2005) 1302-1324.

[28] L. Qi, Hankel tensors: associated Hankel matrices and Vandermonde decomposition, Commun. Math. Sci. 13 (2015) 113-125.

[29] L. Qi, Y. Song, An even order symmetric B tensor is positive definite, Linear Algebra Appl. 457 (2014) 303-312.

[30] L.Q. Qi, F. Wang, Y.J. Wang, Z-eigenvalue methods for a global polynomial optimization problem, Math. Program. 118 (2) (2009) 301-316.

[31] Q. Ni, L. Qi, F. Wang, An eigenvalue method for the positive definiteness identification problem, IEEE Trans. Autom. Control 53 (2008) 1096-1107.

[32] L. Qi, G. Yu, E.X. Wu, Higher order positive semi-definite diffusion tensor imaging, SIAM J. Imag. Sci. 3 (2010) 416-433.

[33] L. Qi, C. Xu, Y. Xu, Nonnegative tensor factorization, completely positive tensors and an hierarchical elimination algorithm, SIAM J. Matrix Anal. Appl. 35 (2014) 1227-1241.

[34] N. Shor, Nondifferentiable Optimization and Polynomial Problems, Kluwer Academic Publications, Bosten, 1998.

[35] S. Solak, D. Bozkurt, Some bounds on $l_{p}$ matrix and $l_{p}$ operator norms of almost circulant, Cauchy-Toeplitz and Cauchy-Hankel matrices, Math. Comput. Appl. Int. J. 7 (3) (2002) 211-218.

[36] S. Solak, D. Bozkurt, On the spectral norms of Cauchy-Toeplitz and Cauchy-Hankel matrices, Appl. Math. Comput. 140 (2) (2003) 231-238.

[37] Y. Song, L. Qi, Some properties of infinite and finite dimension Hilbert tensors, Linear Algebra Appl. 451 (2014) 1-14.

[38] Y. Song, L. Qi, Properties of some classes of structured tensors, J. Optim. Theory Appl. 165 (2015) 854-873.

[39] P. Yuan, L. You, Some remarks on P, $\mathrm{P}_{0}, \mathrm{~B}$ and $\mathrm{B}_{0}$ tensors, Linear Algebra Appl. 459 (2014) 511-521.

[40] Y. Yang, Q. Yang, Further results for Perron-Frobenius theorem for nonnegative tensors, SIAM J. Matrix Anal. Appl. 31 (2010) 2517-2530.

[41] L. Zhang, L. Qi, G. Zhou, M-tensors and some applications, SIAM J. Matrix Anal. Appl. 35 (2014) 437-452. 